\title{
Bibliometric Analysis of Pfizer-BioNTech (BNT162B2): A COVID-19 Vaccine
}

\author{
Waseem Hassan* (iD and Amina Ara \\ Institute of Chemical Sciences, University of Peshawar, Peshawar 25120, Khyber Pakhtunkhwa, Pakistan.
}

\begin{abstract}
The objective of the study was to perform a bibliometric analysis of the Pfizer-BioNTech vaccine. For this purpose Scopus database was used. As of $13^{\text {th }}$ May 2021, one hundred and seventy-three $(n=173)$ research documents are published about it. We used Vosviewer and Biblioshiny (Bibliometrix) in the present report. In all publications, 1160 authors have significantly contributed. The documents per author was $\mathbf{0 . 1 1 9}$, while authors per document was 8.41. Collaborative Index $(\mathrm{Cl})$ was found to be 9.47. By biblioshiny we also applied Lotka's law to depict the author's frequency. 1097 authors were involved in only one $(n=1)$ publication. Based on the Scopus record, the highest documents are published by Dormitzer, P.R. $(n=6)$. In universities and countries categories, Tel Aviv University $(n=7)$ and USA (48) are the most productive. By Vosviewer the collaboration pattern among authors, institutes, and countries is graphically presented. For example, 28 authors, 17 departments and 7 countries directly contributed to one publication. The research documents (133) are published in 102 sources or journals. The highest documents are published in Vaccines $(n=8)$, while the highest citations were recorded for the New England Journal of Medicine $(n=838)$. We also applied co-words analysis to understand the main focus of these publications.
\end{abstract}

Keywords: Bibliometric analysis, COVID-19, Pfizer Vaccine

*Correspondence: waseem_anw@yahoo.com

(Received: May 10, 2021; accepted: June 17, 2021)

Citation: Hassan W, Ara A. Bibliometric Analysis of Pfizer-BioNTech (BNT162B2): A COVID-19 Vaccine. J Pure Appl Microbiol. 2021;15(3):1211-1229. doi: 10.22207/JPAM.15.3.11

(C) The Author(s) 2021. Open Access. This article is distributed under the terms of the Creative Commons Attribution 4.0 International License which permits unrestricted use, sharing, distribution, and reproduction in any medium, provided you give appropriate credit to the original author(s) and the source, provide a link to the Creative Commons license, and indicate if changes were made. 


\section{INTRODUCTION}

Coronavirus disease 2019 (COVID-19), is a highly transmissible global pandemic. It was $1^{\text {st }}$ detected and identified in Wuhan, China, in December 2019. It causes an infection in nose, sinuses, upper throat or lower respiratory tract (windpipe and lungs). Some of the major sympotoms are fever, coughing, shortness of breath, trouble breathing, fatigue, chills, sometimes with shaking, body aches, headache, sore throat, congestion/runny nose, loss of smell or taste, nausea, and diarrhea. The virus can lead to pneumonia, respiratory failure, heart problems, liver problems, septic shock, and ultimately may lead to death. According to WHO, globally, there have been $160,074,267$ confirmed cases of COVID-19, including $3,325,260$ deaths (till $14^{\text {th }}$ May, 2021).

Bibliometrics is the qualitative and quantitative analysis of scientific articles, books, or the chapters of a book. It is widely used for analysis of the research outputs of individual authors, institutes, and countries. It can also help in identifying the impact of research in a particular field. The new and emerging areas of research can also be explored with the help of bibliometrics. It may help to identify the potential research collaborators and find suitable sources or journal in which the research documents can be published ${ }^{1-4}$. However, there are a few issues related with bibliometric analysis. The citation rates vary by subject or field. For example, the top-ranked journals in mathematics have impact factors of around 3; while, in cell biology the journals may have 30 impact factor. Similarly, the $\mathrm{h}$-index varies by field. The life scientists, physicists and social scientists top out at 200, 100 and $20-30$ (h-index), respectively. This indicates the differences between various disciplines. The citation is also database dependent. For example a researcher in Web of Science may have an h-index of 10. But in Google Scholar he/she may have 20 h-index ${ }^{5-7}$.

The scientific literature about COVID-19 has exponentially increased and various bibliometric studies are reported in this regard For example, Mao et al., performed the global bibliometric analysis about the coronavirus research. The authors used Web of Science database and analyzed 9294 articles related with
SARS, MERS and COVID-198. Similarly, Saed H. Zyoud explored the Arab region's contribution to global COVID-19 research. Precisely, they retreived a total of 143,975 publications about COVID-19. The Arab region contribution was $4.25 \%$ or 6131 documents ${ }^{9}$. Its worthy to note that Chinese government has implemented substantial policies to prevent and control the COVID-19 epidemic. Jiang Wu et al., performed bibliometric analysis to understand the transitions and effects of policies over time. A total of 366 policies of epidemic prevention were collected and bibliometrically analyzed ${ }^{10}$. More recently, E. Sachini et al., explored the COVID-19 research publications output of Greek institutions. They identified its advancement over time and analyzed their international collaboration. Bibliometrically, they studied 656 research documents ${ }^{11}$. Similarly, Shaher H. Zyoud and Ahed H. Zyoud performed bibliometric and visualization mapping analysis to understand the hotspots and research trends in COVID-19 and environmental fields. They retrived and studied 729 documents from Scopus database limited to environmental sources only ${ }^{12}$. Kalra, Gagan et al., performed a scientometric analysis of research related with COVID-19 and ophthalmology. They analyzed 616 relevant documents obtained from WoS ${ }^{13}$. While, Ibraín Enrique et al., bibliometrically analyzed 1956 articles about COVID-19 and diabetes ${ }^{14}$. similarly, there are a few more bibliometric studies about COVID-19 to explore the activity and trends ${ }^{15-17}$.

Recently, Cascella et al., provided detail information about the significant progress in the development of novel therapeutics and vaccine development for COVID-19. Currently, a variety of therapeutic options are available which are either approved by U.S. Food and Drug Administration (FDA) (Emergency Use Authorization ( EUA) or being evaluated for COVID-19. For example the anti-SARS-CoV-2 monoclonal antibodies (e.g., bamlanivimab/ etesevimab, casirivimab/imdevimab), antiinflammatory drugs (e.g., examethasone), immunomodulators agents (e.g., baricitinib, tocilizumab) etc. We will specifically highlight the antiviral drugs ${ }^{18}$. Initially Hydroxychloroquine and chloroquine were proposed as antiviral treatments. However according to recent findings the use of hydroxychloroquine with or without 
azithromycin in hospitalized patients did not improve the clinical status or overall mortality compared to placebo ${ }^{19-20}$. Lopinavir/ritonavir is an FDA-approved combo therapy for the treatment of HIV. It was also proposed as antiviral therapy against COVID-19. However a recent data reported no benefit against COVID-19. Currently it is not indicated for the treatment ${ }^{21}$. Ivermectin is an FDA-approved anti-parasitic drug proposed for the treatment of COVID-19. However no significant efficacy was observed in recent trial. Therefore it is currently not indicated for the treatment of COVID-1922. Based on three randomized, controlled clinical trials the FDA approved remdesivir for the treatment of COVID-19. Currently, the SII/Covishield and AstraZeneca/ AZD1222 vaccines were given EUL on 16 February. The Janssen/Ad26.COV 2.S developed by Johnson \& Johnson, was listed for EUL on 12 March 2021. The Moderna COVID-19 vaccine (mRNA 1273) was listed for EUL on 30 April 2021 and the Sinopharm COVID-19 vaccine was listed for EUL on 7 May 2021. While, on December 11, 2020, the Food and Drug Administration (FDA) issued an Emergency Use Authorization (EUA) for the Pfizer-BioNTech COVID-19 Vaccine (also known as BNT162b2, an mRNA vaccine encoding the SARS-CoV-2 spike glycoprotein) for prevention of COVID-19. To the best of our knowledge this is the $1^{\text {st }}$ bibliometric report about Pfizer-BioNTech (vaccine).

\section{MATERIAL AND METHODS \\ Data sources and visualization}

On $13^{\text {th }}$ May, 2021, we retrieved the data from Scopus, one of the largest databases in the world. In search field the following term was used i.e. "Pfizer-BioNTech" OR "BNT162b2". It was searched in article titles, abstracts and keywords i.e. TIT/ABS/KEYWORDS. We quantitatively and qualitatively analyzed in Microsoft Excel 2013. For science mapping and visualization, we used VOSviewer version 1.6.9 and Bibloshiny (Bibliometrix).

\section{RESULTS AND DISCUSSION}

Total one hundred and seventy three $(n=173)$ research documents are published about Pfizer-BioNTech vaccine. It majorly comprised of articles $(n=91)$, reviews $(n=42)$, letters $(n=22)$, notes ( $n=12$ ), editorials ( $n=6)$, short surveys ( $n=4)$ and errata $(n=1)$. For detail analysis we focused on articles and reviews ( $n=133)$ with 1359 citations. One of the advantages of biblioshiny is that it can provide detail information about the local and global citations. Local citations are the citations which are received from documents included in data.While, global citations are the total citations that an article has received from other documents indexed in a database (WOS, Scopus, etc). The local and global citation details of only cited documents are provided in Table 1.

The highest citations are received by three documents which are published in "New England Journal of Medicine". In the $1^{\text {st }}$ report ( $T C=686$ ), the authors performed a multinational, placebo-controlled, observer-blinded, pivotal efficacy trial (conducted in USA and Germany) to test the efficacy of BNT162b2 vaccine. 21, 720 participants were given BNT162b2 and 21, 728 with placebo injections. The author claimed and concluded $95 \%$ efficacy of BNT162b2 in preventing Covid-19.

In the 2 nd most cited document ( $\mathrm{TC}=219$ ), the authors explored the efficacy of two lipid nanoparticle-formulated vaccine candidates (a) BNT162b1 and (b) BNT162b2. Two groups of individuals 18 to 55 years and 65 to 85 years were included in the placebo-controlled, observer-blinded, dose-escalation and phase 1 trial conducted in USA. The author confirmed the safety and immunogenicity data of (BNT162b1) trial conducted in Germany.

In the $3^{\text {rd }}$ highest cited report ( $\left.\mathrm{TC}=51\right)$, the authors obtained data from Clalit Health Services (CHS), the largest health care organization in Israel. recisely, they evaluated and discussed the effectiveness of the BNT162b2 mRNA vaccine. $1,163,534$ vaccinated cases were included in this study along with 596,618 placebo/control subjects. During the period from 14 to 20 days after the first dose, the authors analyzed effectiveness against, documented infection, symptomatic Covid-19, hospitalization, severe disease and death. $46 \%$, $57 \%, 74 \%, 62 \%$ and $72 \%$ effectiveness were (respectively) noted for above four categories. While, during the period from 21 to 27 days after the first dose, the estimated effectiveness for these outcomes was $60 \%, 66 \%, 78 \%, 80 \%$ and 


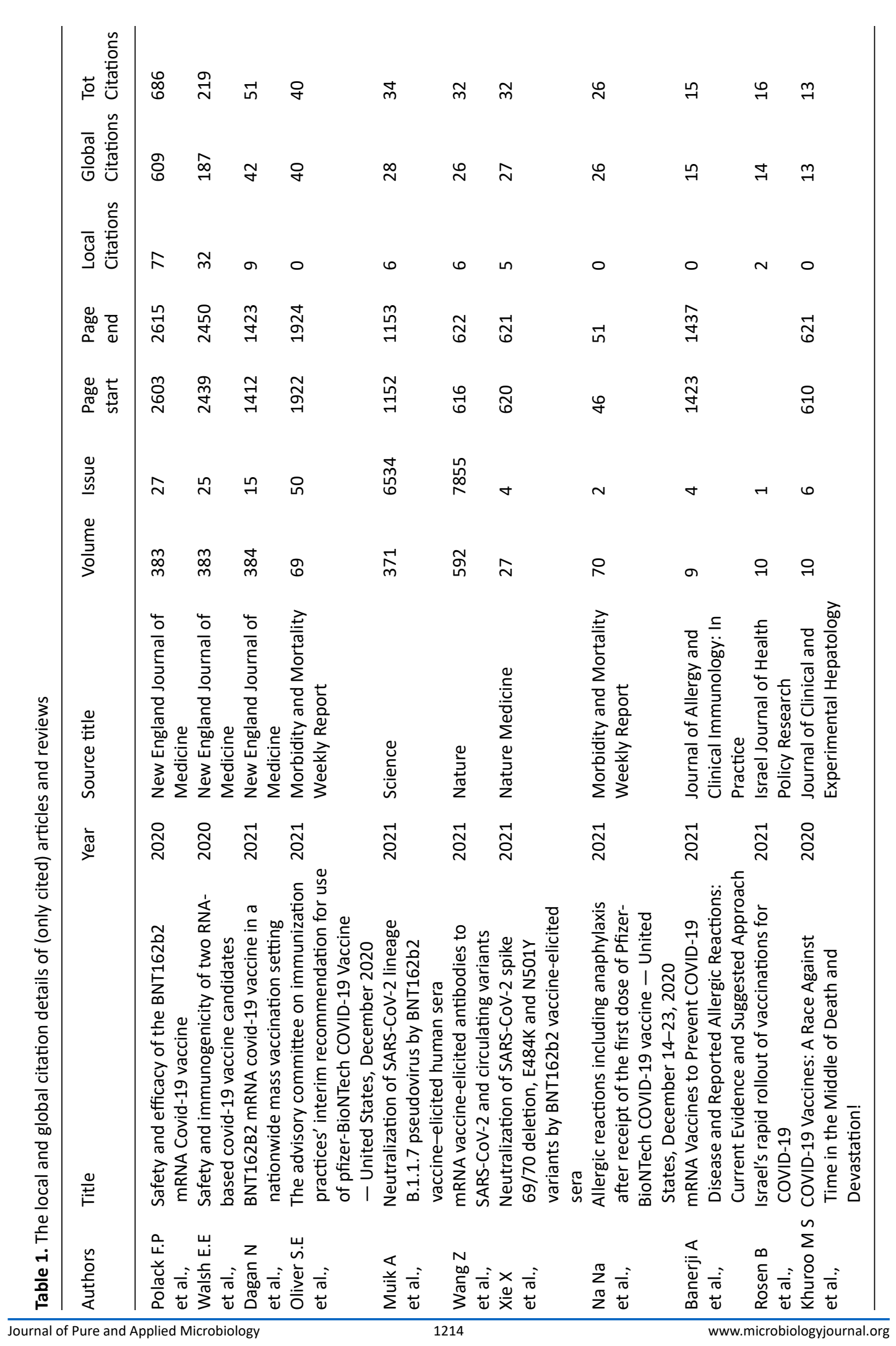




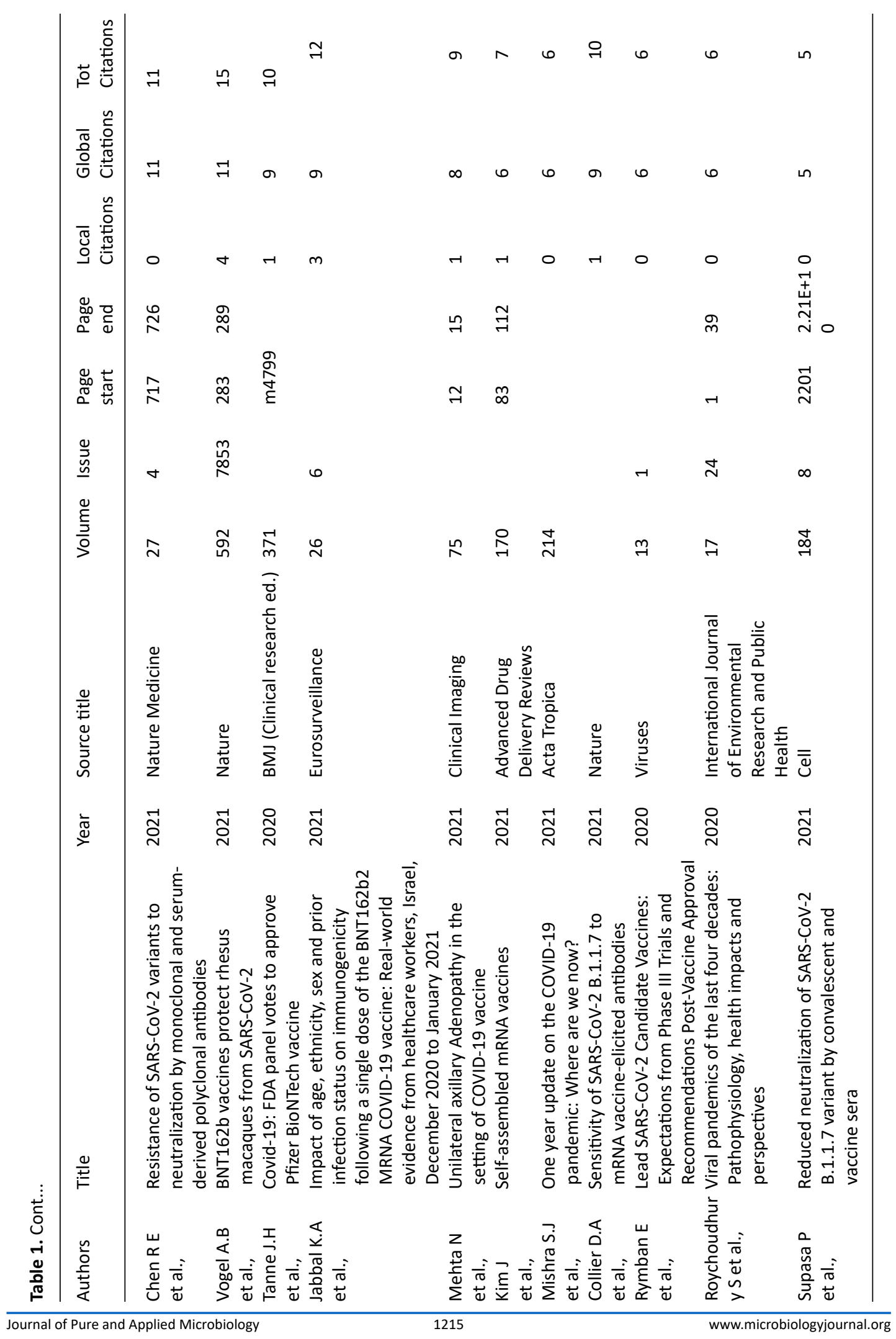




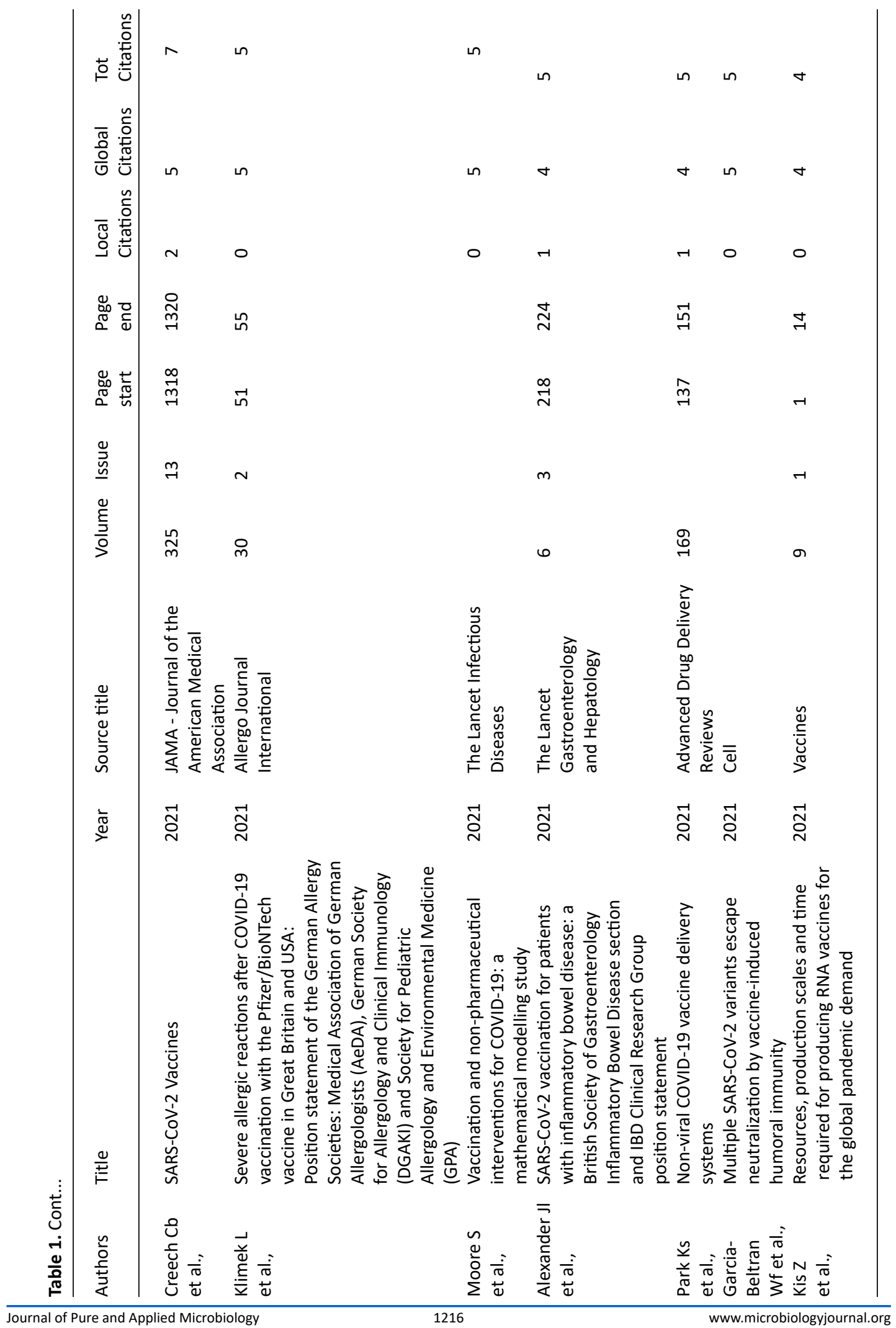




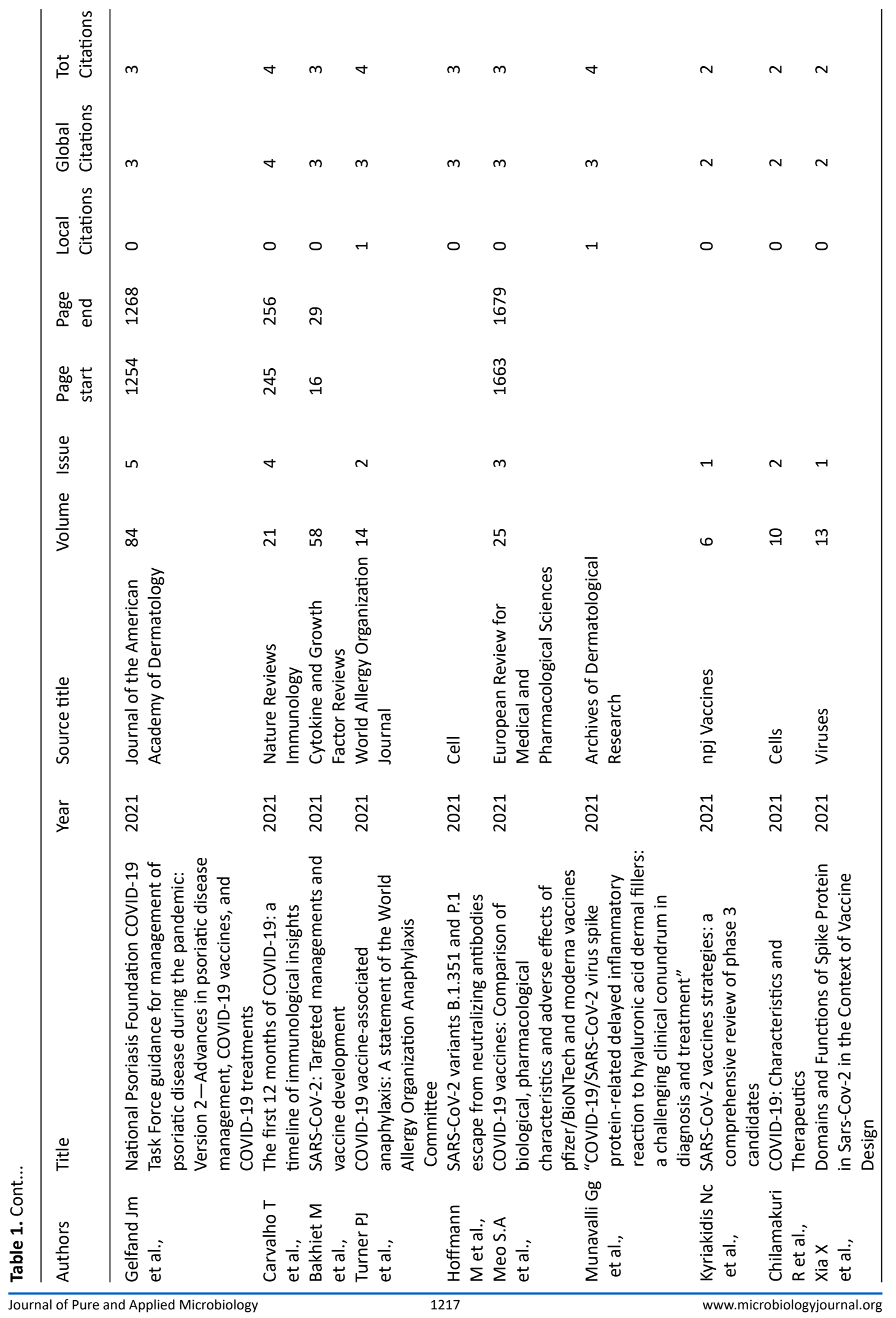




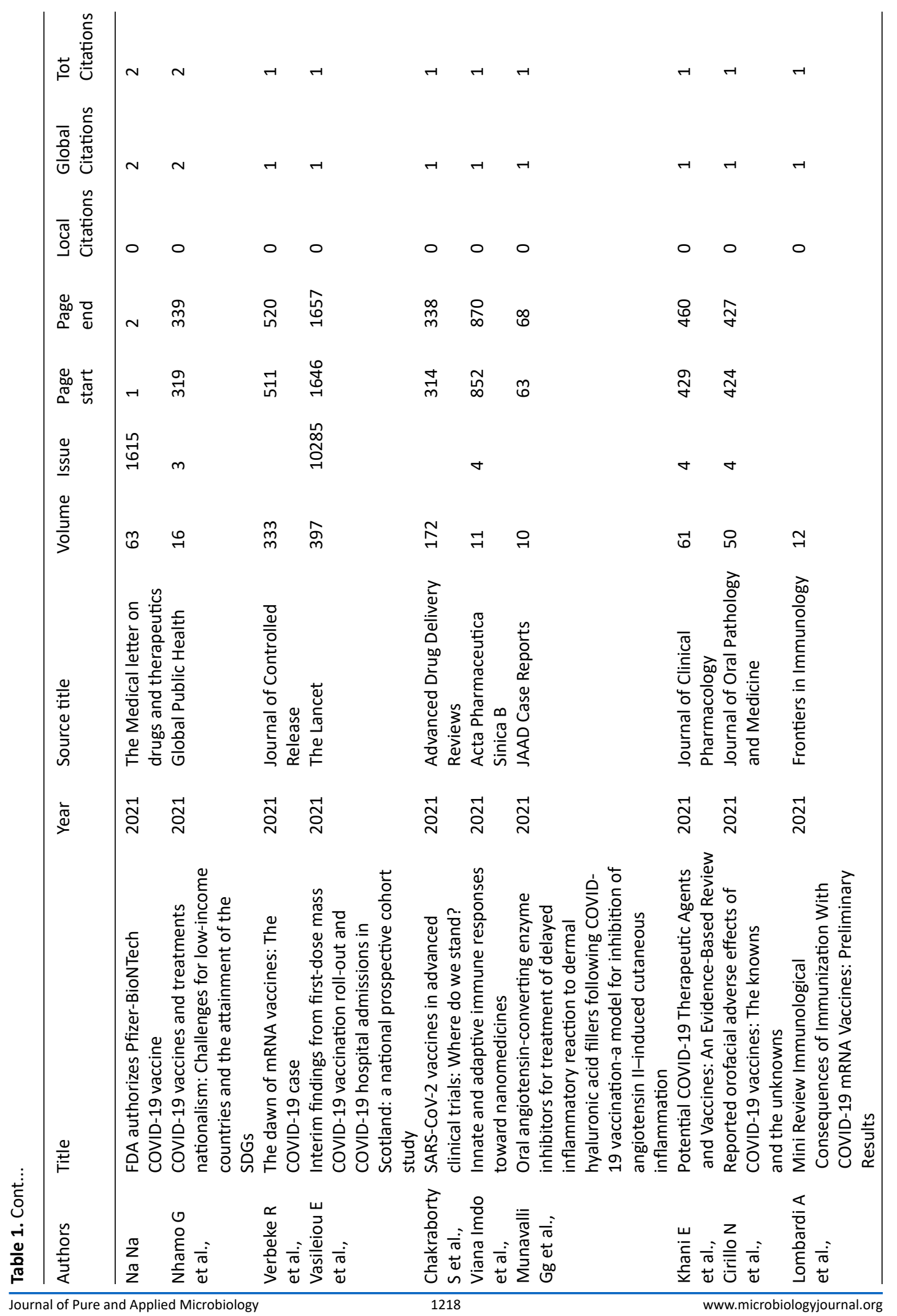




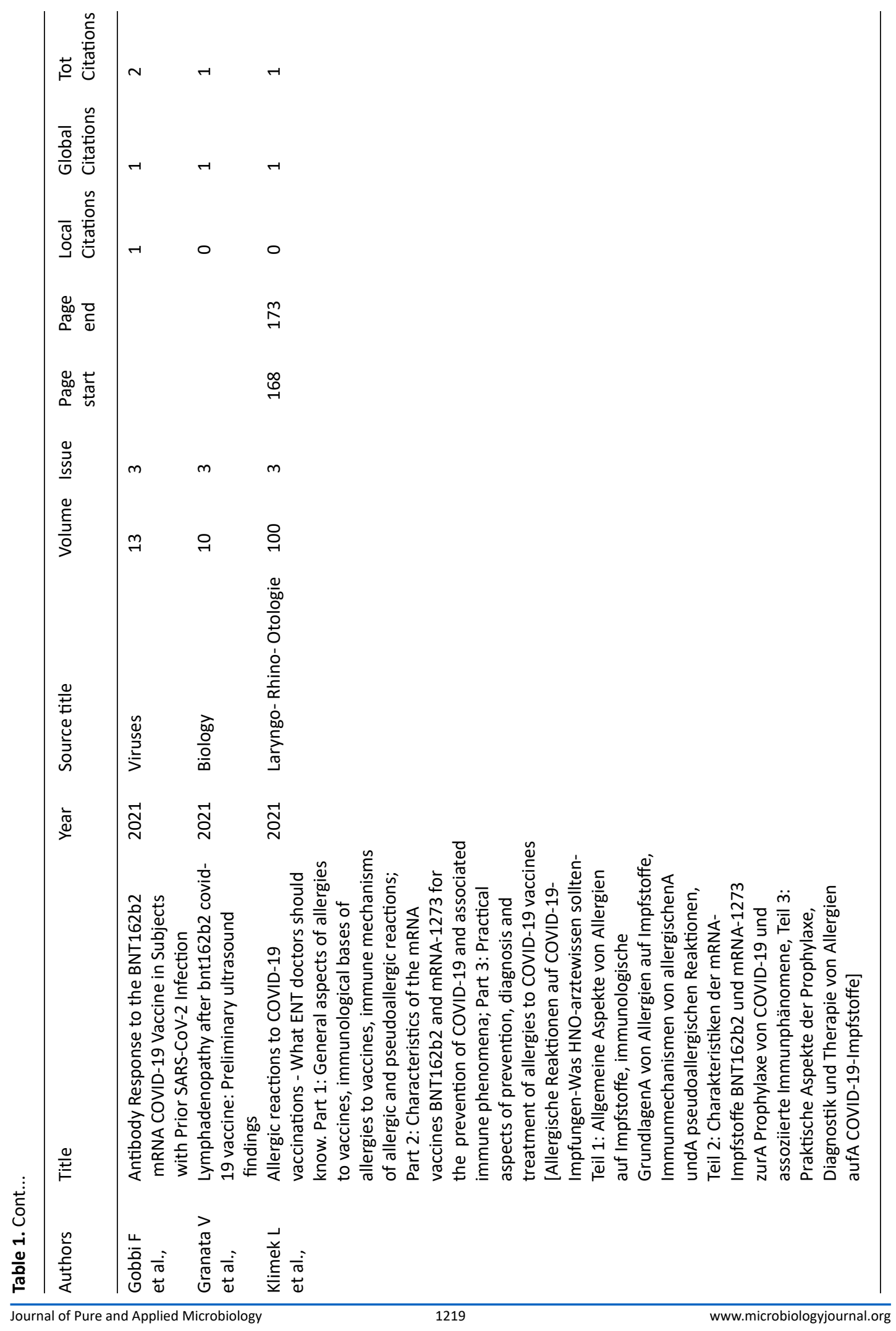




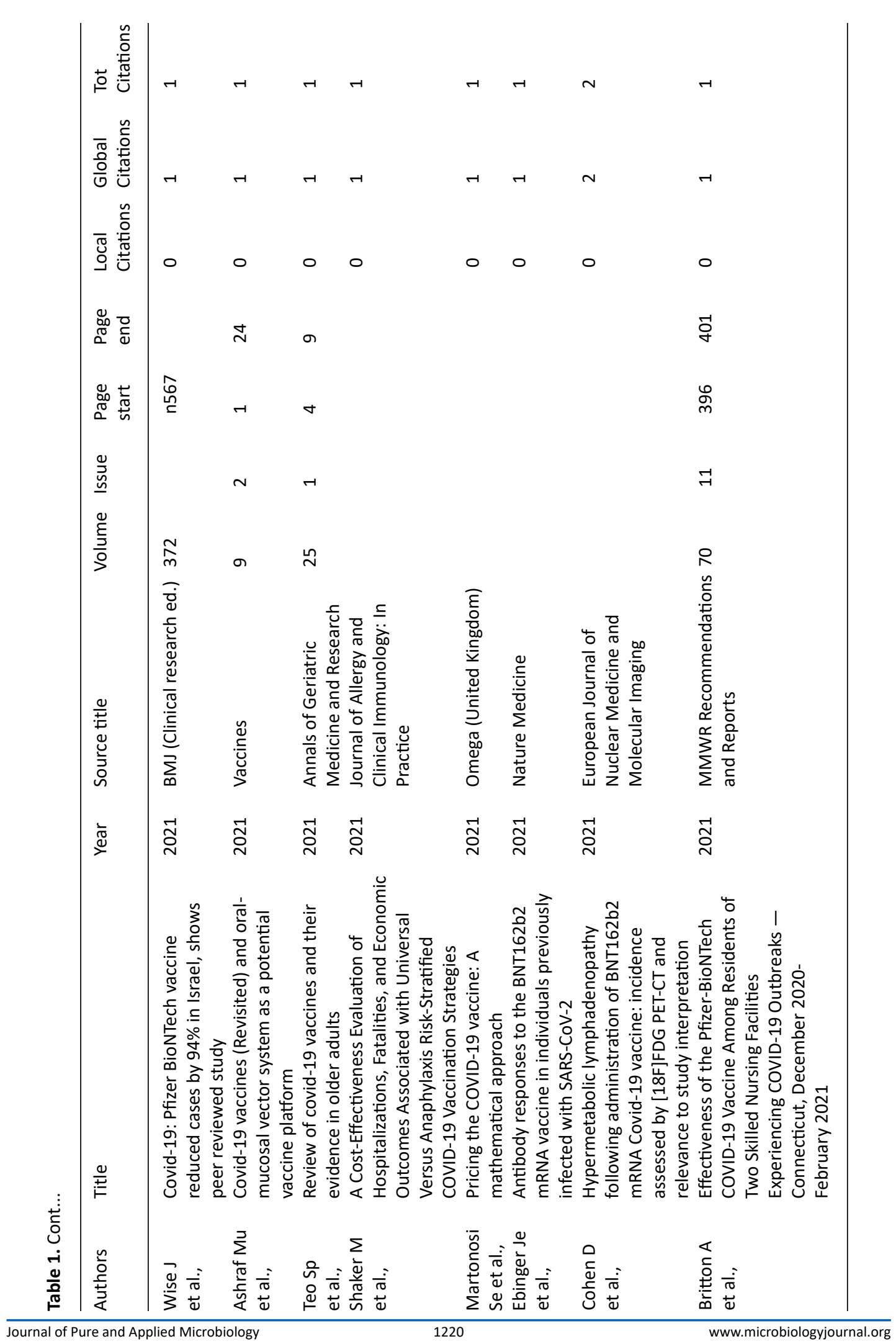




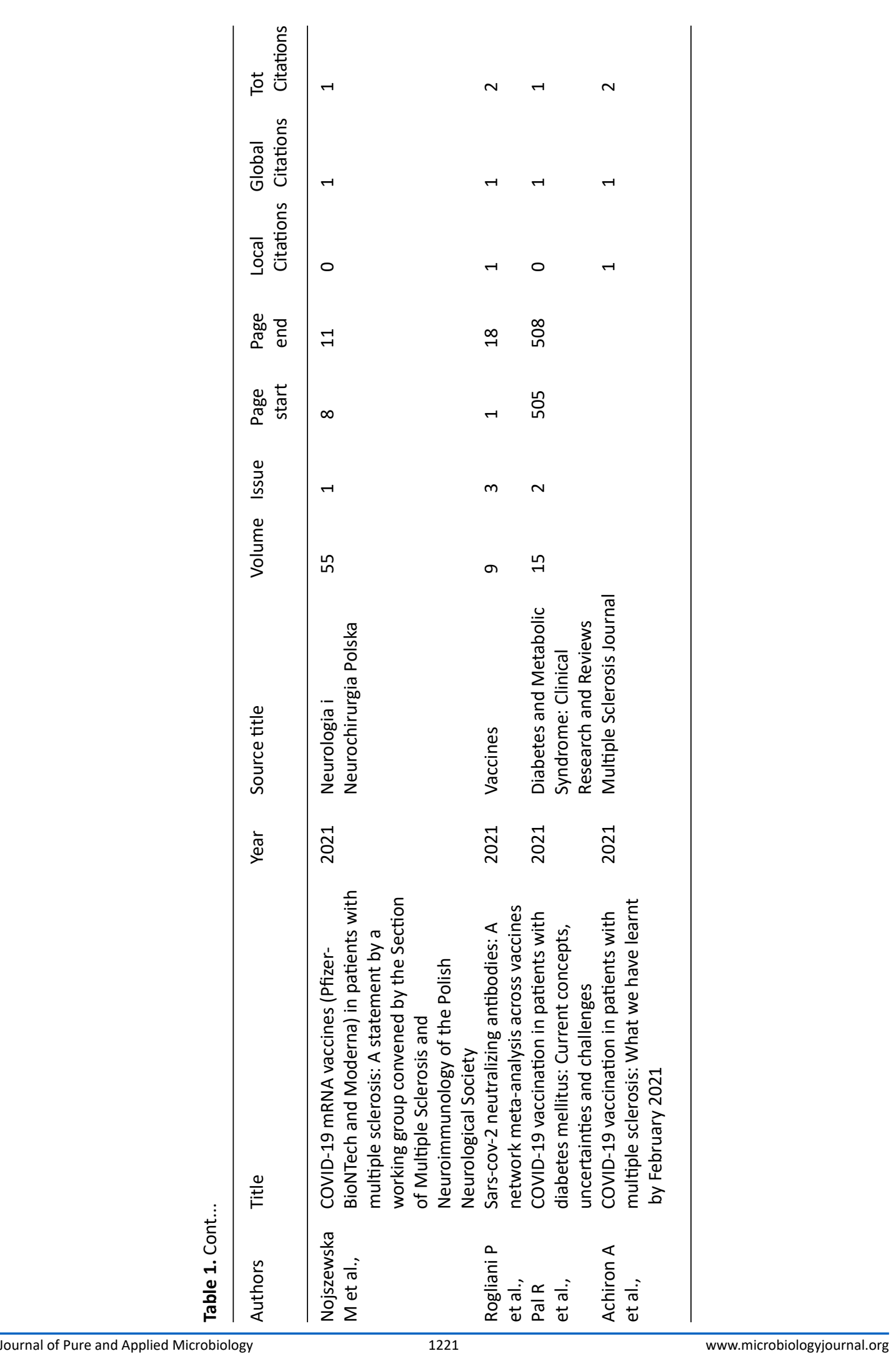


$84 \%$, respectively. Based on the data, the authors suggested that the BNT162b2 mRNA vaccine is effective against Covid-19.

The $4^{\text {th }}$ most cited document is about the summary of "the advisory committee on immunization practices' interim recommendation for use of pfizer-BioNTech COVID-19 Vaccine United States, December 2020". “On December 12, 2020, the Advisory Committee on Immunization Practices (ACIP) issued an interim recommendation for use of the Pfizer-BioNTech COVID-19 vaccine in persons aged $\geq 16$ years for the prevention of COVID-19".

The $5^{\text {th }}$ most cited work $(\mathrm{TC}=34)$ is published in Science. In background, the authors provided brief information about a novel SARSCoV-2 variant, lineage B.1.1.7 discovered in UK. The authors tested efficacy of BNT162b2 (30 $\mu \mathrm{g})$ for neutralization of SARS-CoV-2 Wuhan and lineage B.1.1.7. The data suggested that new line "will not escape" BNT162b2-mediated protection.

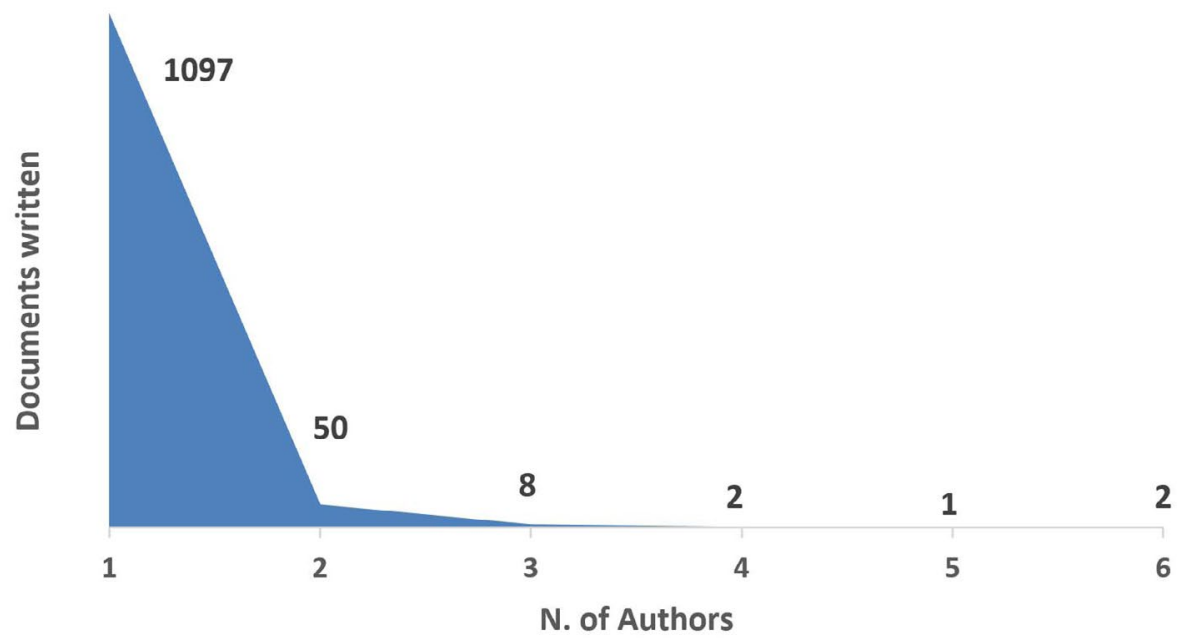

Fig. 1. The authors frequencey by Lotka's law.

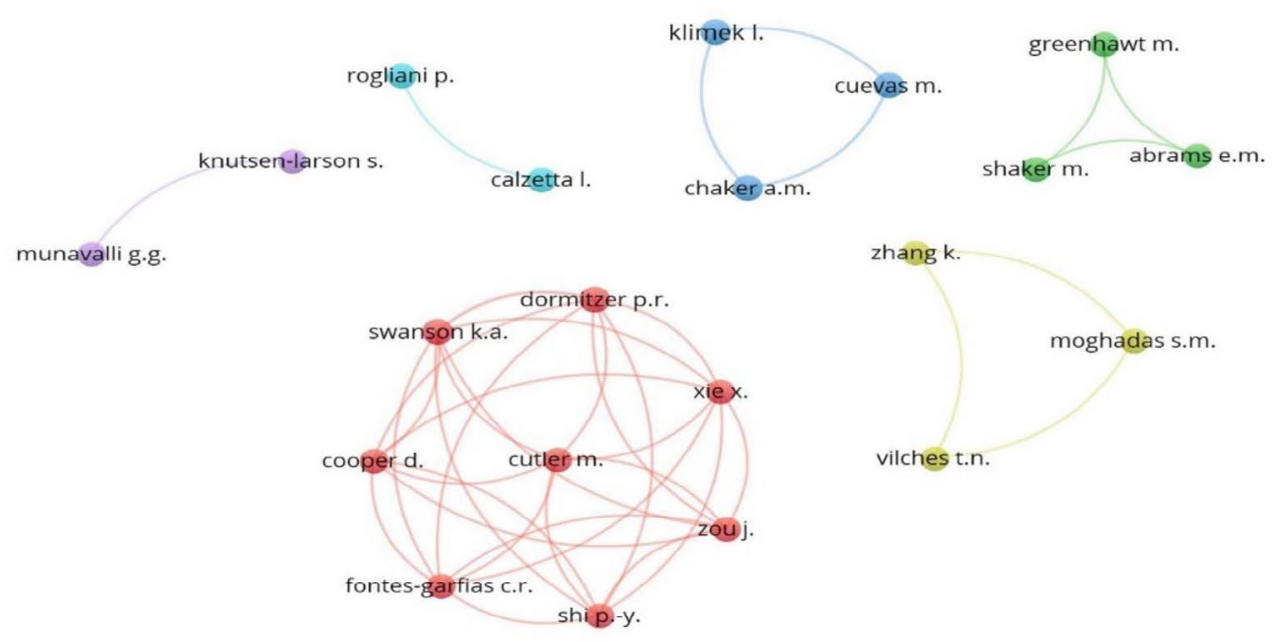

vosviewer

Fig. 2. The co-authorship network for authors. 


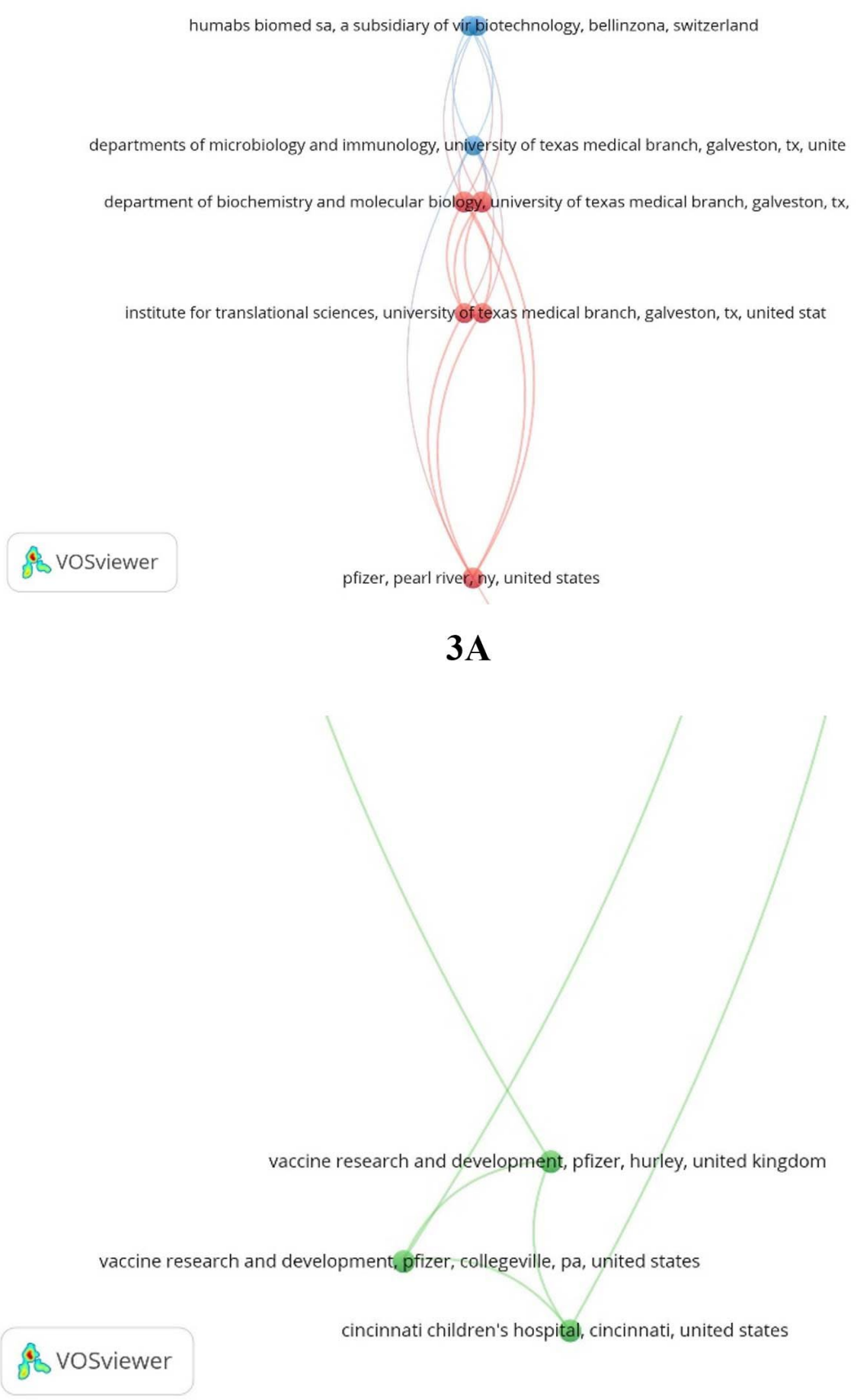

3B

Fig. 3A,B. The co-authorship network for institutes. 
Table 2. The sources/journals details

\begin{tabular}{|c|c|c|c|c|c|c|c|c|}
\hline S\# & Source & NP & ${ }_{\text {index }}^{h_{-}}$ & $\underset{\text { index }}{g_{-}}$ & $\mathrm{m}_{\text {index }}$ & TC & Rank & Zone \\
\hline 1. & New England Journal Of Medicine & 3 & 3 & 3 & 1.5 & 838 & 9 & Zone 1 \\
\hline 2. & Morbidity And Mortality Weekly Report & 3 & 2 & 3 & 2 & 66 & 7 & Zone 1 \\
\hline 3. & Nature & 3 & 3 & 3 & 3 & 46 & 8 & Zone 1 \\
\hline 4. & Nature Medicine & 5 & 2 & 5 & 2 & 39 & 2 & Zone 1 \\
\hline 5. & Science & 1 & 1 & 1 & 1 & 28 & 94 & Zone 3 \\
\hline 6. & $\begin{array}{l}\text { Journal Of Allergy And Clinical Immunology: } \\
\text { In Practice }\end{array}$ & 2 & 1 & 2 & 1 & 16 & 16 & Zone 2 \\
\hline 7. & Israel Journal Of Health Policy Research & 1 & 1 & 1 & 1 & 14 & 58 & Zone 3 \\
\hline 8. & Cell & 3 & 3 & 3 & 3 & 13 & 4 & Zone 1 \\
\hline 9. & $\begin{array}{l}\text { Journal Of Clinical And Experimental } \\
\text { Hepatology }\end{array}$ & 1 & 1 & 1 & 0.5 & 13 & 62 & Zone 3 \\
\hline 10. & Advanced Drug Delivery Reviews & 3 & 2 & 3 & 2 & 11 & 3 & Zone 1 \\
\hline 11. & Bmj (Clinical Research Ed.) & 2 & 1 & 2 & 0.5 & 10 & 11 & Zone 1 \\
\hline 12. & Viruses & 3 & 2 & 3 & 1 & 9 & 10 & Zone 1 \\
\hline 13. & Eurosurveillance & 2 & 1 & 2 & 1 & 9 & 14 & Zone 1 \\
\hline 14. & Clinical Imaging & 1 & 1 & 1 & 1 & 8 & 36 & Zone 2 \\
\hline 15. & Vaccines & 8 & 1 & 2 & 1 & 6 & 19 & Zone 2 \\
\hline 16. & Acta Tropica & 1 & 1 & 1 & 1 & 6 & 21 & Zone 2 \\
\hline 17. & $\begin{array}{l}\text { International Journal Of Environmental } \\
\text { Research And Public Health }\end{array}$ & 1 & 1 & 1 & 0.5 & 6 & 55 & Zone 2 \\
\hline 18. & Allergo Journal International & 1 & 1 & 1 & 1 & 5 & 24 & Zone 2 \\
\hline 19. & $\begin{array}{l}\text { JAMA - Journal Of The American Medical } \\
\text { Association }\end{array}$ & 1 & 1 & 1 & 1 & 5 & 60 & Zone 3 \\
\hline 20. & The Lancet Infectious Diseases & 1 & 1 & 1 & 1 & 5 & 97 & Zone 3 \\
\hline
\end{tabular}

AUTHORS, UNIVERSITIES AND COUNTRIES

Based on biblioshiny (bibliometric) analysis, 1160 authors have contributed to all publications. Fourteen $(n=14)$ authors published single-authored documents and 1146 authors published multi-authored documents. The documents per author was 0.119 , while authors per document was 8.41. Collaborative Index $(\mathrm{Cl})$ can be used as a quantitative measure of research collaboration. It was proosed by Lawani in 1986. It can be calculated by the following formula.

$C=$ Total Number of Authors/ Total Number of Papers

By biblioshiny we calculated the $\mathrm{Cl}$, and it was found to be 9.47

Lotka's Law is normally used to provide breif informaqtion about the frequency of publication of authors in a particular research field. Sometimes it is called "inverse square law", which means that there is an inverse relation between the number of publications and the number of authors. It is depicted by $1 / \mathrm{n}^{2}$. In other words $60 \%$ of authors will have only one publications. $1 / \mathrm{n}^{2} \mathrm{can}$ be further extrapolated, for example, $\left(1 / 2^{2}\right.$ times $60)$ and $\left(1 / 3^{2}\right.$ times 60$)$. This means that $15 \%$ and $7 \%$ authors will have two and three publications, respectively ${ }^{23-24}$. By biblioshiny we applied Lotka's law to depict the authors frequency. Its apparent from the Fig. 1, that 1097 and 50 authors were involved in one $(n=1)$ and two $(n-2)$ publications, respectively.

The highest documents are published by Dormitzer, P.R. $(n=6)$, Swanson, K.A. $(n=6)$, Klimek, L. $(n=5)$, Shi, P.Y. $(n=5)$ and Tureci, O. $(n=4)$. Institutionally, based on the Scopus data, the highest documents were published by Tel Aviv University $(n=7)$, followed by Pfizer Inc. $(n=6)$, UT Medical Branch at Galveston $(n=5)$, Imperial College London ( $n=5)$, Universita degli Studi di Milano $(n=5)$ and BioNTech SE $(n=5)$. While, Harvard Medical School, Tel Aviv University, Sackler Faculty of Medicine, University of Toronto, Technical University of Munich, Klinikum rechts der Isar der Technischen Universitat Munchen and Zentrum Allergie und Umwelt all published four $(n-4)$ documents. 
Total 46 countries were directly involved in all publications. The highest documents were published by United States ( $n=48)$, Germany $(n=22)$, United Kingdom ( $n=17)$, Italy $(n=16)$ and Canada $(n=13)$. Other countries in the list includes

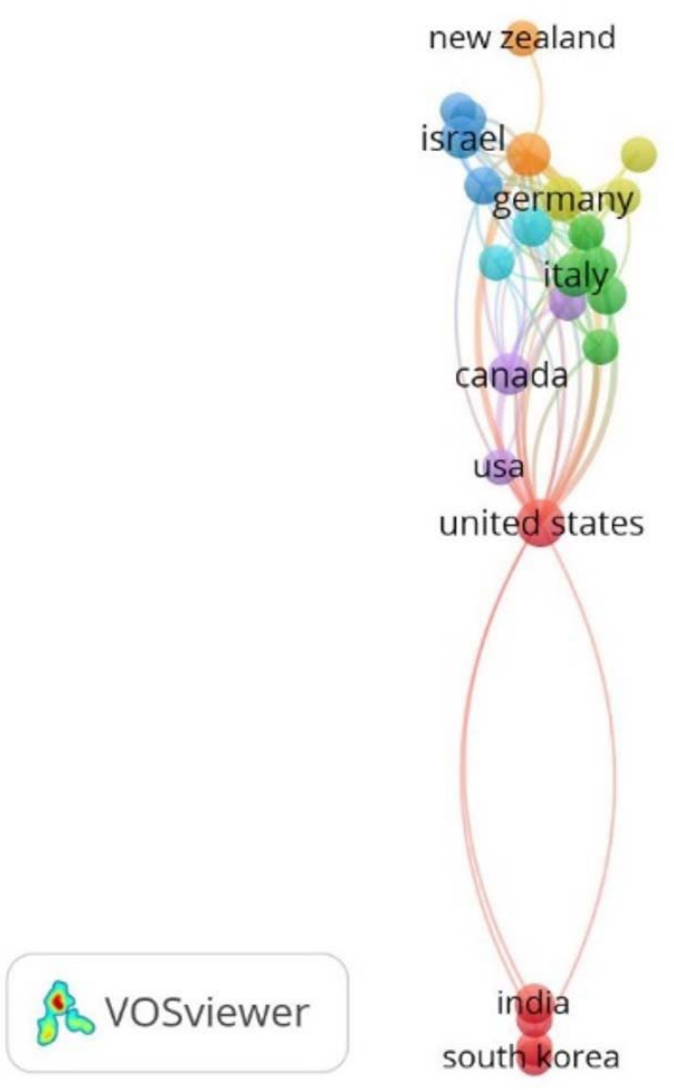

Fig. 4. The co-authorship network for countries.
Israel ( $N=12)$, India $(n=8)$, South Africa $(n=6)$, Spain $(n=6)$, Brazil $(n=5)$, Australia $(n=4)$ and Netherlands $(n=4)$.

The collaboration among authors, institutes, and countries could be explained by the fact that;

1. 28 authors (Polack F.P., Thomas S.J., Kitchin N., Absalon J., Gurtman A., Lockhart S., Perez J.L., Marc G.P., Moreira E.D., Zerbini C., Bailey R., Swanson K.A., Roychoudhury S., Koury K., Li P., Kalina W.V., Cooper D., Frenck R.W., Hammitt L.L., Tureci O., Nell H., Schaefer A., Ünal S., Tresnan D.B., Mather S., Dormitzer P.R., Sahin U., Jansen K.U., Gruber W.C., C4591001 Clinical Trial Group;

2. From 17 departments or hospitals (Fundacion INFANT, Buenos Aires, Argentina; ITrialsHospital Militar Central, Buenos Aires, Argentina; State University of New York, Upstate Medical University, Syracuse, NY, United States; Vaccine Research and Development, Pfizer, Pearl River, NY, United States; Vaccine Research and Development, Pfizer, Hurley, United Kingdom; Vaccine Research and Development, Pfizer, Collegeville, PA, United States; Worldwide Safety, Safety Surveillance and Risk Management, Pfizer, Collegeville, PA, United States; Associacao Obras Sociais Irma Dulce and Oswaldo Cruz Foundation, Bahia, Brazil; Centro Paulista de Investigacao Clinica, Sao Paulo, Brazil; Global Product Development, Pfizer, Peapack, NJ, United States; Cincinnati Children's Hospital, Cincinnati, United States; Johns

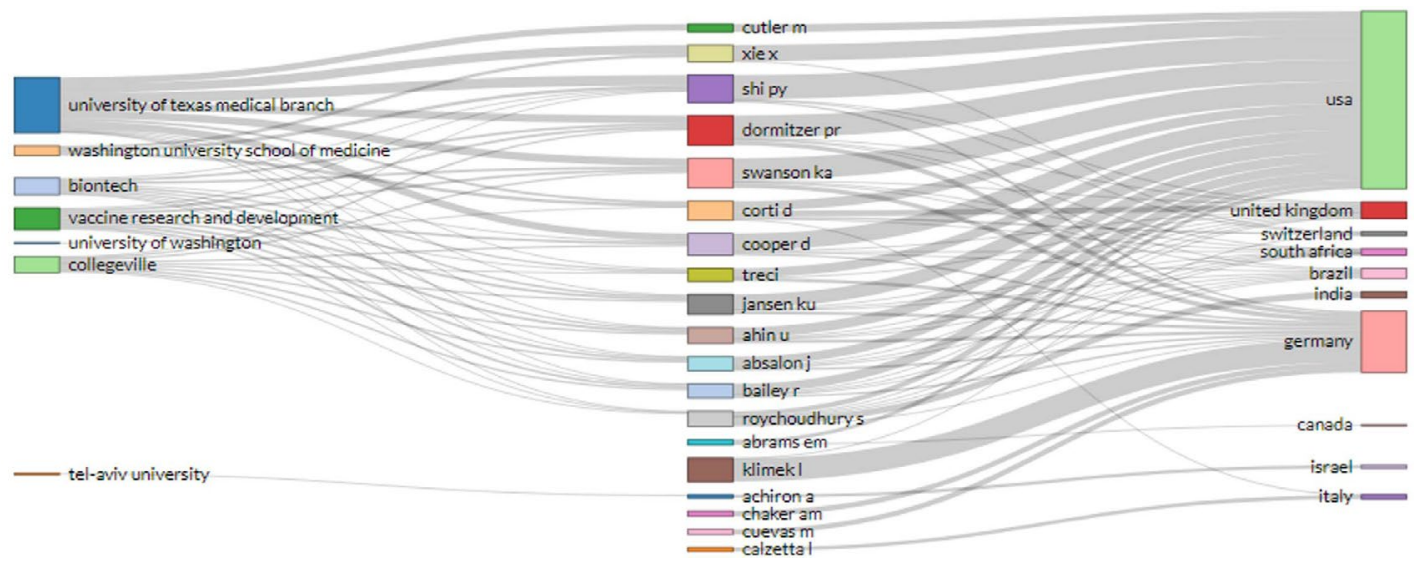

Fig. 5. The co-authorship network for authors, institutes and countries (three fields plot). 
Hopkins Bloomberg School of Public Health, Baltimore, United States; BioNTech, Mainz, Germany; Medizentrum Essen Borbeck, Essen, Germany; Tiervlei Trial Centre, Karl Bremer
Hospital, Cape Town, South Africa; Hacettepe University, Ankara, Turkey; Worldwide Safety, Safety Surveillance and Risk Management, Pfizer, Groton, CT, United States)

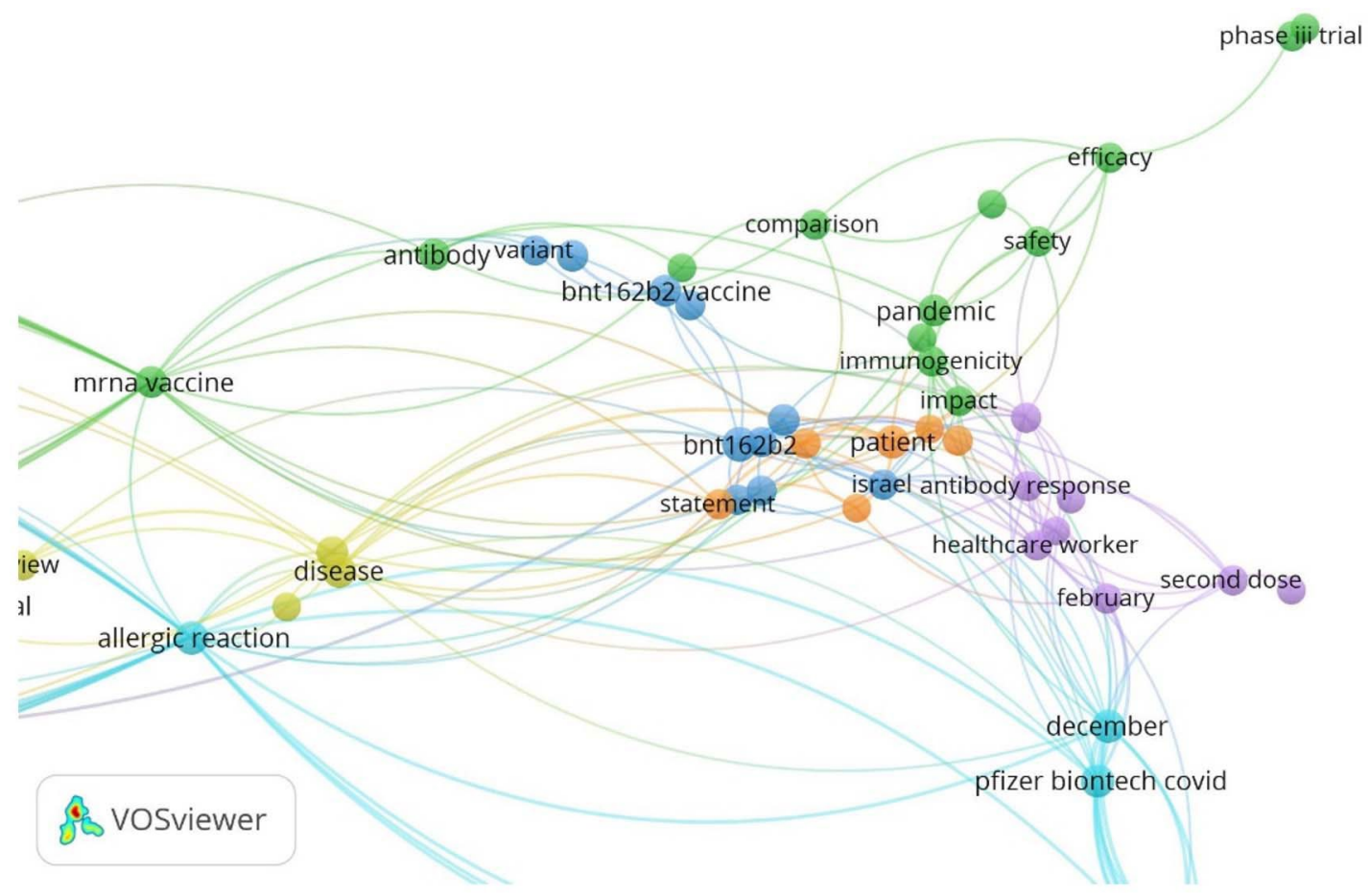

6A

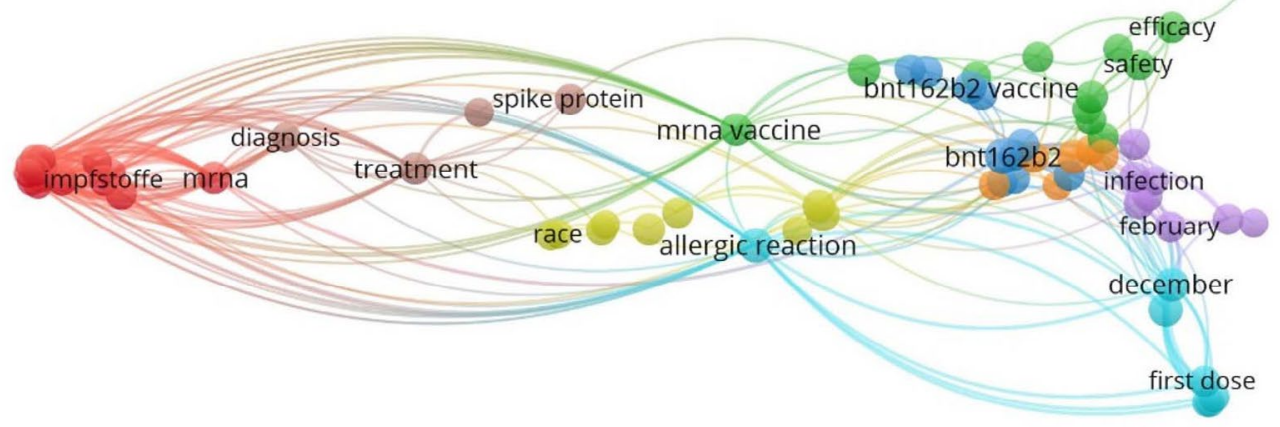

\& Vosviewer

\section{B}

Fig. 6. The co-words analysis of publications. 
3. From 7 countries (Argentina, Brazil, USA, UK, Germany, South Africa and Turkey) collectively contributed to a single article published in New England Journal of Medicine.

By Vosviewer we also presented the collaboration between those authors who have published atleast two documents. The data is depicted in Fig. 2. For example, in red cluster there are total eight authors. They collectively contributed to the following research documents.

1. Safety and efficacy of the BNT162b2 mRNA Covid-19 vaccine

2. Safety and immunogenicity of two RNA-based covid-19 vaccine candidates

3. Neutralization of SARS-CoV-2 lineage B.1.1.7 pseudovirus by BNT162b2 vaccine-elicited human sera

4. Neutralization of SARS-CoV-2 spike $69 / 70$ deletion, E484K and N501Y variants by BNT162b2 vaccine-elicited sera

5. Resistance of SARS-CoV-2 variants to neutralization by monoclonal and serumderived polyclonal antibodies

6. BNT162b vaccines protect rhesus macaques from SARS-CoV-2

Interestingly these authors were from different institutes as shown in Fig. $3 \mathrm{~A}$ and B. Furthermore, twenty-six countries have published atleast two research documents. Their interconnectivity is described in Fig. 4.

While, by three fields plot we also represented authors, institutes, and countries in the same diagram (Fig. 5).

Sources

The documents are published in 102 sources. The highest documents are published in Vaccines $(n=8)$ and Nature Medicine $(n=5)$. However, the highest citations were noted for New England Journal Of Medicine ( $n=838)$, followed by Morbidity And Mortality Weekly Report $(n=66)$ and Nature $(n=46)$. The list of top 20 sources with $\mathrm{H}$-index, g-index and $\mathrm{m}$-index details are described in table 2.

\section{Co-words Analysis}

Based on the co-words analysis it was noted that the publications focused on clinical trials (Phase 1, 2 and 3 Clinical Trial). Different subject groups were included (young to aged) in reports. The drug designing, safety, administration, dosage, side effects of bnt162b1 and bnt162b2 was explored for the possible treatment of COVID-19. The authors also analyzed various symptoms associated with COVID i.e. fatigue, fever, headache, inflammation, infection, injection site pain, and mortality (caused by). The genetic \& immune response and epidemiology was also studied in detail. The co-words are described in Fig. 6 A \&B.

Limitations

One of the major limitations of the present study is that we only explored Scopus. Other common databases, such as PubMed, Embase, and WoS, were not searched. Thus, some influential papers may have been missed. Therefore, future bibliometric studies with other databases are recommended which may complement the present findings. We also did not analyze letters, notes, editorials, conference papers etc. Most of the articles are recently published. Ample time is required to get proper citations. Consequently, it may affect the rankings of the manuscript (based on citations). Citation analysis may have spelling, names changes, homonyms, clerical errors, addition of post codes, language biases and problem with journal impact factors. However we did not consider it in the present report. Furthermore, we did not analyze self-citations, which may affect the total citation count.

\section{CONCLUSION}

There are various bibliometric reports about the COVID-19. To the best of our knowledge, this is the first study in which we summarized the publication histroy of the Pfizer-BioNTech vaccine. Initially, we discussed the advantages and disadvantage of bibliometric studies. Later some information about the novel therapeutics and vaccine development for COVID-19 is added. Based on the Scopus record, one hundred and seventy three $(n=173)$ research documents (91 articles \& 42 reviews) are published about Pfizer vaccine. Based on biblioshiny analysis, the local and global citation record is provided. We also briefly discussed the top five cited documents. 1160 authors have significantly contributed to all publications. The collaborative index $(\mathrm{Cl})$ was found to be 9.47. By Lotka's Law, we provided breif informaqtion about the frequency of publication of authors. For example, 1097 authors contributed 
to only one publication. Based on the number of publication, details about the top authors, university and country is provided. By Vosviewer analysis we presented the collaboration between authors, institutes, and countries. The documents are published in 102 sources. The citations details (h-index, g-index and m-index) of the top 20 sources are also provided. Last but not the least, the trend and focus of publication is depicted by co-words analysis.

\section{ACKNOWLEDGMENTS}

We sincerely appreciate the hard work of all researchers, institutes and countries involved for combating this global pandemic.

\section{CONFLICT OF INTEREST}

The authors declare that there is no conflict of interest.

\section{AUTHORS' CONTRIBUTION}

Both the authors have made a substantial, direct and intellectual contribution to the work, and approved it for publication.

\section{FUNDING}

None.

\section{DATA AVAILABILITY}

The data is retrieved from the Scopus database.

\section{ETHICS STATEMENT}

Not applicable.

\section{REFERENCES}

1. Lin WY. Research status and characteristics of library and information science in Taiwan: a bibliometric analysis. Scientometrics. 2012;92(1):7-21. doi: 10.1007/s11192-012-0725-6

2. Zhuang $Y$, Liu $X$, Nguyen $T$, He $Q$, Hong S. Global remote sensing research trends during 1991-2010: a bibliometric analysis. Scientometrics. 2013;96(1):203219. doi: 10.1007/s11192-012-0918-z

3. Huffman MD, Baldridge A, Bloomfield GS, et al. Global cardiovascular research output, citations, and collaborations: a time-trend, bibliometric analysis (1999-2008). PLoS One. 2013;8(12):e83440. doi: 10.1371/journal.pone.0083440

4. White HD, McCain KW. Visualizing a discipline: An author co-citation analysis of information science, 1972-1995. J Am Soc Inf Sci. 1998;49(4):327-355. doi: 10.1002/(SICI)1097-4571(19980401)49:4<327::AID-
ASI4>3.0.CO;2-4

5. Waltman L, Calero-Medina C, Kosten J, et al. The Leiden Ranking 2011/2012: Data collection, indicators, and interpretation. J Am Soc Inf Sci. 2012;63(12):24192432. doi: 10.1002 /asi.22708

6. Hirsch JE. An index to quantify an individual's scientific research output. Proc Natl Acad Sci USA. 2005;102(46):16569-16572. doi: 10.1073/ pnas.0507655102

7. Bar-llan J. Which h-index?-A comparison of WoS, Scopus and Google Scholar. Scientometrics. 2008;74(2):257271. doi: 10.1007/s11192-008-0216-y

8. Mao X, Guo L, Fu P, Xiang C. The status and trends of coronavirus research: A global bibliometric and visualized analysis. Medicine. 2020;99(22):e20137. doi: 10.1097/MD.0000000000020137

9. Sa'ed $\mathrm{HZ}$. The Arab region's contribution to global COVID-19 research: Bibliometric and visualization analysis. Globalization and Health. 2021;17(1):31. doi: 10.1186/s12992-021-00690-8

10. Wu J, Wang K, He C, Huang X, Dong K. Characterizing the patterns of China's policies against COVID-19: A bibliometric study. Information Processing \& Management. 2021;58(4):102562. doi: 10.1016/j. ipm.2021.102562

11. Sachini E, Sioumalas-Christodoulou K, Chrysomallidis C, Siganos G, Bouras N, Karampekios N. COVID-19 enabled co-authoring networks: a country-case analysis. Scientometrics. 2021;126(6): 5225-5244. doi: 10.1007/s11192-021-03952-9

12. Zyoud SH, Zyoud AH. Coronavirus disease-19 in environmental fields: a bibliometric and visualization mapping analysis. Environment, Development and Sustainability. 2021;23:8895-8923. doi: 10.1007/ s10668-020-01004-5

13. Kalra G, Kaur R, Ichhpujani P, Chahal R, Kumar S. COVID-19 and ophthalmology: A scientometric analysis. Indian J Ophthalmol. 2021;69(5):1234-40. doi: 10.4103/ijo.IJO_3284_20

14. Corrales-Reyes IE, Hernandez-Garcia F, Mejia CR. COVID-19 and diabetes: Analysis of the scientific production indexed in Scopus. Diabetes \& Metabolic Syndrome: Clinical Research \& Reviews. 2021;15(3):765-770. doi: 10.1016/j.dsx.2021.03.002

15. Fan J, Gao Y, Zhao N, et al. Bibliometric analysis on COVID-19: a comparison of research between English and Chinese studies. Front Public Health. 2020;8:477. doi: 10.3389/fpubh.2020.00477

16. DE Felice F, Polimeni A. Coronavirus Disease (COVID-19): A Machine Learning Bibliometric Analysis. In Vivo. 2020;34(3):1613-1617. doi: 10.21873/ invivo.11951

17. Yu Y, Li Y, Zhang Z, et al. A bibliometric analysis using VOSviewer of publications on COVID-19. Ann Transl Med. 2020;8(13):816. doi: 10.21037/atm-20-4235

18. Cascella M, et al. Features, Evaluation, and Treatment of Coronavirus (COVID-19). 2021 Apr 20. In: StatPearls [Internet]. Treasure Island (FL): StatPearls Publishing. PMID: 32150360 Free Books \& Documents. https:// pubmed.ncbi.nlm.nih.gov/32150360/

19. Horby $P$, Mafham M, Linsell L, et al. Effect of Hydroxychloroquine in Hospitalized Patients with 
COVID-19: Preliminary results from a multi-centre, randomized, controlled trial. MedRxiv. 2020. doi: 10.1101/2020.07.15.20151852

20. Zhang R, Mylonakis E. In inpatients with COVID-19, none of remdesivir, hydroxychloroquine, lopinavir, or interferon $\beta$-1a differed from standard care for inhospital mortality. Ann Inter Med. 2021;174(2):JC17. doi: 10.7326/ACPJ202102160-017

21. Cao B, Wang $\mathrm{Y}$, Wen D, et al. A trial of lopinavirritonavir in adults hospitalized with severe Covid-19. N Engl J Med. 2020;382(19):1787:1799. doi: 10.1056/
NEJMoa2001282

22. Lopez-Medina E, Lopez P, Hurtado IC, et al. Effect of ivermectin on time to resolution of symptoms among adults with mild COVID-19: a randomized clinical trial. JAMA. 2021;325(14):1426-1435. doi: 10.1001/ jama.2021.3071

23. Lawani S. Some bibliometric correlates of quality in scientific research. Scientometrics. 1986;9(1-2):13-25. doi: 10.1007/BF02016604

24. Potter WG. Lotka 's Law Revisited. Library Trends, 1981;30(1):21-40. 